\title{
One-Step Protein Purification: Use of a Novel Epitope Tag for Highly Efficient Detection and Purification of Recombinant Proteins
}

\author{
Stefan Rasche ${ }^{1}$, Alexander Martin ${ }^{2}$, Achim Holzem ${ }^{3}$, Rainer Fischer ${ }^{1,3}$, Helga Schinkel ${ }^{1}$ and \\ Stefan Schillberg*,1 \\ ${ }^{I}$ Fraunhofer Institute for Molecular Biology and Applied Ecology (IME), Forckenbeckstraße 6, 52074 Aachen, Germany \\ ${ }^{2}$ Aachen University of Applied Sciences, Heinrich-Mussmann-Str, 52428 Jülich, Germany. \\ ${ }^{3}$ RWTH Aachen University, Institute for Molecular Biotechnology, Worringer Weg 1, 52074 Aachen, Germany
}

\begin{abstract}
We used the interaction between an epitope of the Tobacco mosaic virus 54K replicase (tag54) and its corresponding monoclonal antibody 54 (mAb54) for the design of a new epitope-tagging system. We fused the DNA sequence of tag54 and two elongated derivates thereof to the C-terminus of the chloramphenicol acetyltransferase (CAT) gene and produced the tagged proteins in tobacco. Immunoblot and ELISA analysis demonstrated that the tagged proteins were detected with high sensitivity in plant extracts. Moreover, the tag54 system enabled the full recovery of the recombinant CAT with excellent purity through immunoaffinity chromatography.
\end{abstract}

We consider this novel epitope-tag system to be a valuable tool for both the detection and purification of recombinant proteins.

Keywords: Epitope tag, immunoaffinity, protein purification, tag54, tobacco.

\section{INTRODUCTION}

Protein purification is one of the most crucial steps in the production of recombinant proteins. Commonly used purification strategies involve several steps, such as ion exchange, hydrophobic interaction or size exclusion chromatography, in order to obtain a pure product. Such a multistep strategy is time-consuming and involves a considerable loss of product, resulting in high production costs. A one-step method, which would obviate these problems, is therefore desirable. One of the most popular methods to circumvent these problems utilizes immobilized metal affinity chromatography to purify recombinant proteins containing a small polyhistidine tag. These tagged proteins interact with immobilized metal ion residues such as nickel or copper [1]. A major drawback of this method is that histidine-containing host-cell proteins copurify with the desired product. This results in a low purification efficiency of the target protein, so that in most cases additional purification steps are required. As an alternative approach, immunoaffinity purification can be employed. This method is based on the specific interaction between a monoclonal antibody $(\mathrm{mAb})$ and its corresponding epitope. Fusing the DNA sequence of the epitope as a tag to the gene for a recombinant protein allows for the highly specific and sensitive detection and purification of the tagged protein [24]. While epitope tags are commonly used in prokaryotic systems without major restrictions, their use in eukaryotes has shown that the detecting antibody can cross-react with endogenous proteins [5-7]. A high-performance tagging

*Address correspondence to this author at the Fraunhofer Institute for Molecular Biology and Applied Ecology (IME), Forckenbeckstraße 6, 52074 Aachen, Germany; Tel: +49-241-6085-11050; Fax: +49-241-6085-10000; E-mail: stefan.schillberg@ime.fraunhofer.de system that could be used for the detection and purification of recombinant proteins in plants would be a significant advance with respect to the growing use of plants as research and application tools.

\section{MATERIALS AND METHODOLOGY}

\section{Generation, Production and Purification of mAb54}

$\mathrm{BALB} / \mathrm{c}$ mice were immunized with purified $54 \mathrm{~K}$ protein from TMV $(3 \times 40 \mu \mathrm{g})$. The spleen cells were then isolated, and hybridoma cells were generated. Selection led to a cell line producing a monoclonal antibody (mAb54) that recognized the antigen [8]. MAb54 were produced in RPMI (Invitrogen, Karlsruhe, Germany) medium containing fetal calf serum and then purified using protein A.

\section{Peptide Display}

Two peptide libraries, pVIII [9] and pVIII9aaCys [10], were used in M13 phage to determine the epitope sequence of the purified mAb54. After 3 panning rounds against $20 \mu \mathrm{g}$ mAb54, 20 phage were sequenced and the result was aligned to the amino acid sequence of the $54 \mathrm{~K}$ protein.

\section{Surface Plasmon Resonance}

The affinity constant between mAb54 and the three differently-sized epitope tags was determined by surface plasmon resonance using a BIAcore T100 system (Biacore, Uppsala, Sweden) [11]. A polyclonal, rabbit anti-mouse $\mathrm{Fc}_{\gamma^{-}}$ specific antibody was immobilized onto the surface of a CM5 sensor chip using an amine coupling kit (Biacore).

Serial dilutions of the peptides (synthetic 12-tag54, 8tag54 and 6-tag54 peptides, JPT, Berlin, Germany) ranging from $160-1.25 \mathrm{nM}$ were measured to determine the kinetic 
parameters of the interaction. In the first step, 950-1000 Response units $(\mathrm{Ru}) \mathrm{mAb5} 4$ were captured $(0.15 \mu \mathrm{l} / \mathrm{min}$, $4 \mathrm{~min}$ ), followed by the injection of one peptide dilution. The binding experiments used a flow rate of $30 \mu \mathrm{l} / \mathrm{min}(180 \mathrm{sec}$ association/240 sec dissociation) at $25^{\circ} \mathrm{C}$ in HBS-EP buffer (10 mM Hepes, pH 7.4, $150 \mathrm{mM} \mathrm{NaCl}, 3.4 \mathrm{mM}$ EDTA, $0.005 \%(\mathrm{v} / \mathrm{v})$ Tween 20). The chip surface was regenerated with $2 \times 15 \mu 130 \mathrm{mM} \mathrm{HCl}$ after each binding experiment. A reference flow cell was used for background subtraction. The data was evaluated using the Biacore T100 evaluation software (version 1.1.1). A 1:1 binding model was used to determine the kinetic parameters.

\section{Construction of Plant Expression Vectors}

Three differently-sized epitope tags were deduced from the consensus sequence that was derived from peptide display: 6-tag54 (KDWEHL), 8-tag54 (IKDWEHLE), and 12tag54 (KHIKDWEHLEEF). CAT (kindly provided by Dr. Hirsch, Institute for Biology I, RWTH Aachen University, Germany) was amplified by PCR, adding the DNA-sequence for each of the three epitope tags to the C-terminus using overlapping primers. The three PCR-products were inserted into pTRAkt, a derivative of pPAM (GenBank accession AY027531). This resulted in the constructs pTRAkt-CAT6tag54, pTRAkt-CAT8-tag54 and pTRAkt-CAT12-tag54, respectively (Fig. 1). All of the constructs were sequenced for verification.

\section{Protein Expression}

The plant expression vectors that had been generated were introduced into Agrobacterium tumefaciens using the Gene Pulser II electroporation system (Bio-Rad, München, Germany) according to the manufacturer's instructions. Agrobacteria containing the different constructs were used for the transient transformation of detached leaves of Nicotiana tabacum cv. Petite Havana SR1 via vacuum infiltration [12]. After infiltration, the leaves were incubated on moist paper in a sealed tray for $60 \mathrm{~h}\left(18 / 6\right.$ light/dark cycle, $23^{\circ} \mathrm{C}$, 5000-10000 lux).

\section{SDS-PAGE and Immunoblot Analysis}

The samples were mixed with SDS loading buffer and electrophoresed using $15 \%(\mathrm{w} / \mathrm{v})$ SDS-Page. After running the gel, it was stained with either Coomassie blue or silver nitrate, or the proteins were transferred onto a nitrocellulose membrane by semidry electrotransfer (125 mA/gel, $25 \mathrm{~min})$.
In the latter case, the membrane was incubated in PBS supplemented with $0.05 \%(\mathrm{v} / \mathrm{v})$ Tween $20(\mathrm{PBS}-\mathrm{T})$ and $5 \%$ $(\mathrm{w} / \mathrm{v})$ skim milk for $30 \mathrm{~min}$ after the transfer.

CAT was detected using $2 \mu \mathrm{g}$ rabbit anti-CAT serum (Sigma-Aldrich, München, Germany) $/ \mathrm{ml}$ in PBS-T. $60 \mathrm{ng}$ alkaline phosphatase-labeled goat anti-rabbit antibody (Jackson ImmunoResearch, West Grove, USA) per $\mathrm{ml}$ in PBS-T was used as the secondary antibody. To detect recombinant

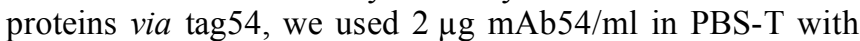
$60 \mathrm{ng}$ alkaline phosphatase-labeled goat anti-mouse antibody/ml (GAM-AP, Jackson ImmunoResearch) in PBS-T as the secondary antibody. All immunoblots were developed for one minute using NBT/BCIP as a substrate. CAT-tag54 was quantified using densitometric measurements of the signals on the immunoblot. A CAT standard (Roche, Mannheim, Germany) series (5-20 ng) was loaded next to four different dilutions of the sample. By comparing the intensity of the bands of the standard series to the samples (AIDA, RayTest, Straubenhardt, Germany), we were able to calculate the concentration of CAT in the samples.

\section{Quantification of Native CAT-tag54}

We detected and quantified native CAT-tag54 in total soluble protein (TSP) using the CAT ELISA kit (Roche), according to the manufacturer's instructions.

\section{Quantification of mAb54}

A high-binding ELISA plate (Greiner Bio-One $\mathrm{GmbH}$, Frickenhausen, Germany) was coated with $60 \mathrm{ng}$ goat-Fab anti-mouse $\mathrm{Fab} / \mathrm{ml}$ in PBS (Jackson ImmunoResearch) for $1 \mathrm{~h}$ at room temperature. All further incubation steps were also performed for the same time and at room temperature. Between all incubation steps, the ELISA plate was washed twice with PBS-T. The plates were blocked with $5 \%(\mathrm{w} / \mathrm{v})$ skim milk in PBS-T. Several dilutions of mAb54, ranging from $1: 500$ to $1: 128,000$, were loaded onto the ELISA plate next to a serial dilution of a standard antibody (mouse antihuman; Jackson ImmunoResearch) ranging from 200 to $0.2 \mathrm{ng} / \mathrm{ml}$. The antibodies were detected using $120 \mathrm{ng}$ goat anti-mouse Fc-specific antibody/ml (Jackson ImmunoResearch) labeled with horseradish peroxidase (HRPO), with ABTS as the substrate.

\section{Immobilization of mAb54}

Mab54 was immobilized onto an NHS-activated sepharose HiTrap Column (GE Healthcare, Freiburg, Germany)

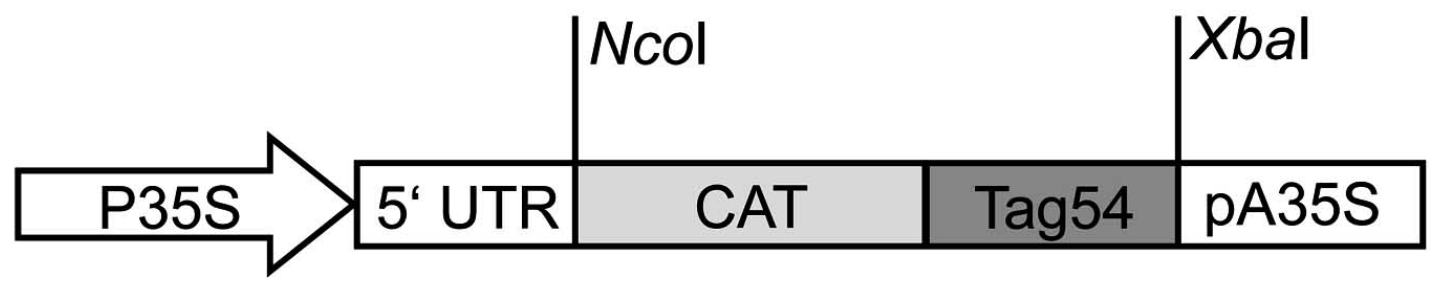

Fig. (1). Construction of the expression cassette for the production of tagged CAT.

The DNA sequences for 6-tag54, 8-tag54 and 12-tag54 were fused to the 3'end of the CAT-gene via PCR. The original stop codon of the CAT gene was exchanged for a codon coding for the amino acid serine and a stop codon was added after the tag54. The expression cassette was cloned into the vector pTRAkt.

P35S: double-enhanced Cauliflower mosaic virus (CaMV) 35S promoter; 5' UTR: 5' untranslated region from Tobacco etch virus; pA35S: CaMV 35S-terminator; tag54*: 12-tag54: 5' AAA CAC ATC AAA GAC TGG GAA CAC CTG GAA GAA TTC 3', 8-tag54: 5' ATC AAA GAC TGG GAA CAC CTG GAA 3', 6-tag54: 5' AAA GAC TGG GAA CAC CTG 3'. 
Table 1. CAT-tag54 Quantification Using Immunoblotting and ELISA

\begin{tabular}{|c|c|c|c|}
\hline & CAT6-tag54 & CAT8-tag54 & CAT12-tag54 \\
\hline \hline Immunoblot & $90.3 \pm 22.9 \mu \mathrm{g} / \mathrm{g}$ & $82.5 \pm 21.2 \mu \mathrm{g} / \mathrm{g}$ & $127.1 \pm 34.8 \mu \mathrm{g} / \mathrm{g}$ \\
\hline ELISA & $115.8 \pm 22.0 \mu \mathrm{g} / \mathrm{g}$ & $111.2 \pm 23.0 \mu \mathrm{g} / \mathrm{g}$ & $149.6 \pm 69.2 \mu \mathrm{g} / \mathrm{g}$ \\
\hline
\end{tabular}

CAT-tag54 concentrations per gram of leaf (fresh weight) were determined using immunoblotting and ELISA. Total CAT-tag54 concentrations were determined using immunoblotting, while native CAT-tag54 was detected using ELISA. The values listed are the means and standard deviations, $\mathrm{n}=3$.

according to the manufacturer's instructions. To determine the coupling efficiency, samples were taken during the immobilization reaction and quantified via ELISA. The antibody matrix was stored at $4^{\circ} \mathrm{C}$ in storage buffer $(50 \mathrm{mM}$ $\left.\mathrm{Na}_{2} \mathrm{HPO}_{4} \mathrm{pH} 7.4,0.1 \%(\mathrm{w} / \mathrm{v}) \mathrm{NaN}_{3}\right)$ until further use.

\section{Protein Purification}

The total soluble protein (TSP) was extracted from infiltrated tobacco leaves by grinding them in liquid nitrogen. A volume of extraction buffer, equal to twice the fresh weight was added and the suspension was centrifuged $\left(45 \mathrm{~min} / 4^{\circ} \mathrm{C} / 108,000 \mathrm{~g}\right)$, filtered $(0.45 \mu \mathrm{m})$ and stored on ice. The buffers were degassed before use and stored on ice during the purification. After equilibration with five volumes of wash buffer (200 mM Tris HCl, pH 7.4, 5 mM EDTA, $0.1 \%$ (v/v) Tween 20), the TSP was loaded at $0.7 \mathrm{ml} / \mathrm{min}$. The column was washed until the $\mathrm{OD}_{280}$ baseline was reached. The tagged protein was eluted using glycine buffer $(50 \mathrm{mM}$ glycine $\mathrm{HCl}, \mathrm{pH} 2.5,150 \mathrm{mM} \mathrm{NaCl}$ ) at $0.3 \mathrm{ml} / \mathrm{min}$, and $1 \mathrm{ml}$ fractions were collected. The TSP and all purification fractions (flow through, wash, elution) were analyzed using immunoblotting and ELISA to determine the amount of tagged protein in these samples.

\section{Determination of the Detection Limit}

To determine the detection limit of denatured CATtag54, samples ranging from $8-1 \mathrm{ng}$ were analyzed by immunoblotting. The samples were detected with mAb54 and the detection limit was defined as the concentration of the last band that was visible in the dilution series. The detection limit for native CAT-tag54 used a modified protocol for the

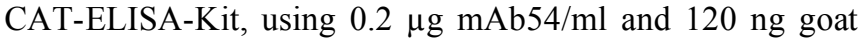
anti-mouse labeled with alkaline phosphatase $/ \mathrm{ml}$ as the primary and secondary antibodies, respectively. The samples were loaded at seven dilutions ranging from $100-1 \mathrm{ng} / \mathrm{well}$. The detection limit was defined as the concentration of the last sample in the dilution series that gave a signal $\mathrm{OD}_{405 \mathrm{~nm}} \geq 0.1$ after background subtraction.

\section{RESULTS AND DISCUSSION}

\section{Epitope Mapping of mAb54}

Peptide display technology was used to determine the epitope corresponding to mAb54 [13]. After three panning rounds, 80 binders were analyzed via ELISA to determine their reactivity against mAb54. The 20 best binders were sequenced (data not shown), and the consensus sequence was compared to the sequence of $54 \mathrm{~K}$ replicase. The epitope to mAb54 was KDWEHL. Next to this corresponding epitope, two additional versions of tag54, 8-tag54 (IKDWEHLE) and 12-tag54 (KHIKDWEHLEEF) were designed, containing an additional two or four epitope flanking amino acids, respectively.

\section{Characterization of the Different Tag Versions}

To compare the productivity of tobacco leaves for the three fusion proteins CAT6tag54, -8tag54 and -12tag54, and to obtain information about the sensitivity of the protein detection by mAb54, tobacco leaves were transiently transformed using A. tumefaciens. The TSP was extracted from the leaves three days after infiltration, and the native and total CAT-tag54 concentrations were measured (Table 1). No clear differences in protein accumulation were detected between the three constructs. The average accumulation level was independent of the tag54 size and was approximately $100 \pm 31 \mu \mathrm{g}$ per $\mathrm{g}$ leaf fresh material, as measured by immunoblotting (ELISA: $126 \pm 42 \mu \mathrm{g} / \mathrm{g}$ ). The discrepancies between the measurements are due to the higher sensitivity of ELISA compared to immunoblotting.

The detection limits for the leaf extracts were determined using immunoblotting and ELISA to obtain data for total and native CAT-tag54. With both methods, the same ratio between the size of the tag54 version and the detection limit was observed (Table 2). The shorter the tag 54 sequence, the lower the sensitivity, even though the epitope identified by peptide display (KDWEHL) was unchanged. It is possible that the identified epitope is displaced within the entire 12 amino acid-long tag54 sequence, or that flanking amino acids influence the folding of tag54, resulting in increased sensitivity to detection by mAb54. Additional experiments, including an alanine scan [14], will be performed to elucidate the role of the flanking amino acids. Neither degradation bands nor cross-reactivity with endogenous plant proteins were detectable on any immunoblot (data not shown).

Table 2. Detection Limit of tag54 in Immunoblots and ELISA

\begin{tabular}{|c|c|c|}
\hline & Immunoblot $[\mathbf{n g} / \mathbf{b a n d}]$ & ELISA $[\mathbf{n g} / \mathbf{m l}]$ \\
\hline \hline CAT6-tag54 & $4.3 \pm 1.5$ & $80 \pm 8^{*}$ \\
\hline CAT8-tag54 & $2.4 \pm 1.4$ & $13 \pm 2$ \\
\hline CAT12-tag54 & $1.0 \pm 0.1$ & $11 \pm 5$ \\
\hline
\end{tabular}

Immunoblots were developed for $1 \mathrm{~min}$, and ELISA was developed for $20 \mathrm{~min}$, after adding the substrate solution. With decreasing tag size, the sensitivity decreased, independently of whether CAT-tag54 was in its native or denatured form. The values listed are the means and standard deviations, $n=3$, except for $* n=2$.

Small tags are not likely to have an impact on the folding and functionality of the target protein and therefore their removal is not required, as it is often the case for larger tags like the maltose binding protein or glutathione S-transferase tag [4]. A comparison of our results with those from commercially-available tagging systems demonstrates the advantages of the tag54 system. For example, 8-tag54 (which only has eight amino acids) is detectable down to $1.2 \mathrm{ng}$ per band in immunoblot analysis, and is therefore as sensitive as the 
8-amino acid FLAG-tag (Sigma-Aldrich). The 12-tag54 (detection limit $<1 \mathrm{ng}$ per band) is clearly superior to the p24 tag [15], which has 11 amino acids and a detection limit of $2.2 \mathrm{ng}$ per band. Moreover, this already high sensitivity could be improved using multiple copies of the tags and a different substrate for detection [16].

The kinetic parameters of the interaction between the three synthetic tag54 variants and mAb54 were determined by surface plasmon resonance (Fig. 2). The 12-tag54 and the 8-tag54 peptides show comparable kinetic parameters, whereas the 6-tag54 peptide has approximately $90 \%$ reduced affinity to mAb54 (Table 3). The decrease in the affinity confirms the data of the previous detection limit analysis (Table 2). Based upon these results, we decided to focus on the 12-tag54 in further experiments.
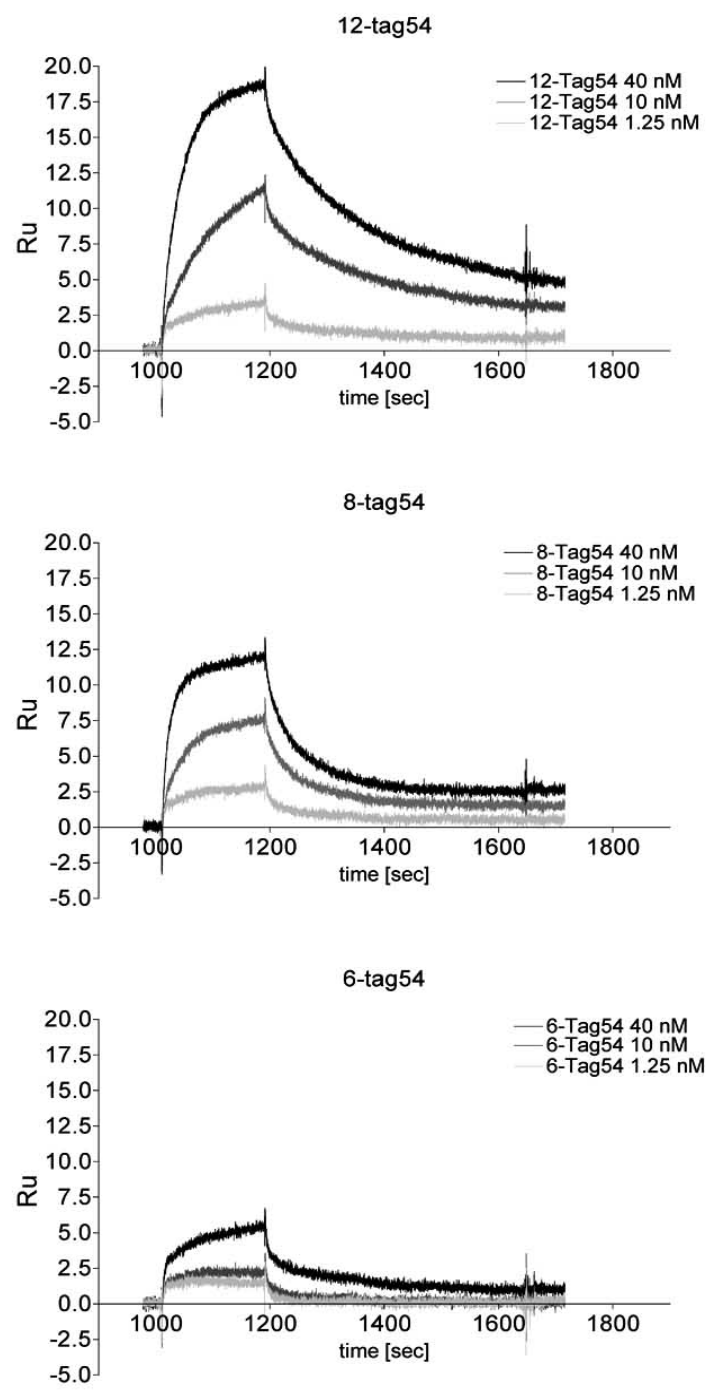

Fig. (2). Surface plasmon resonance analysis of tag54 variants.

The kinetic parameters of the interaction between the three synthetic tag54 peptide variants and the mAb54 were determined by SPR. The mAb54 was immobilized on the surface of a CM5 chip and different concentrations of each peptide, ranging from $160-$ $1.25 \mathrm{nM}$, were measured to determine the kinetic parameters. Here the measurements of three representative concentrations $(40 \mathrm{nM}$, $10 \mathrm{nM}$ and $1.25 \mathrm{nM}$ ) for each peptide are shown.
Table 3. SPR Analyses

\begin{tabular}{|c|c|c|c|}
\hline Peptide & $\mathbf{k a}\left(\mathbf{M}^{-1} \mathbf{s}^{-1}\right)$ & kd (1/s) & KD(M) \\
\hline \hline 12-tag54 & $7.2^{*} 10^{5}$ & $3.7 * 10^{-3}$ & $5.2^{*} 10^{-9}$ \\
\hline 8-tag54 & $1.5^{*} 10^{6}$ & $8.8^{*} 10^{-3}$ & $6.0^{*} 10^{-9}$ \\
\hline 6-tag54 & $8.8^{*} 10^{4}$ & $3.1 * 10^{-3}$ & $3.6^{*} 10^{-8}$ \\
\hline
\end{tabular}

The kinetic parameters for the three peptide tags were determined by surface plasmon resonance. Serial dilutions of each peptide ranging from $160-1.25 \mathrm{nM}$ were used. A $1: 1$ binding model was used to fit the parameters. ka: association rate, kd: dissociation rate, KD: equilibrium constant.

Nevertheless, even the 6-tag 54 has a higher affinity to the mAb54 than the FLAG-tag has to the anti-FLAG antibody M1 (KD $\left.4,1 * 10^{-7} \mathrm{M}\right)$. The FLAG/anti-FLAG system is a comparable commercial system (Sigma Aldrich) that is frequently used for the purification of recombinant proteins by immunoaffinity chromatography [17].

\section{Affinity Purification of Epitope-Tagged Proteins}

Prior to protein purification, a mAb54 immunoaffinity matrix was prepared. The coupling efficiency of the mAb54 to the NHS-activated Sepharose was determined by quantifying the mAb54 concentration via ELISA before and after the coupling reaction. An efficiency of $99.5 \%$ was reached after $60 \mathrm{~min}$. A total of $15 \mathrm{mg}$ of $\mathrm{mAb} 54 / \mathrm{ml}$ of matrix was immobilized, giving a maximal column capacity of $200 \mathrm{nmol}$ of tagged protein. The antibody was immobilized in a nondirectional fashion, resulting in the partially inaccessible presentation of mAb54. The actual column capacity is therefore estimated to be approximately $30 \%$ of the theoretical column capacity [18]. This did not restrict the work presented here, as larger amounts of CAT-tag54 were not purified. However, in future experiments, a directed immobilization method (e.g. CarboLink, Thermo Scientific) that results in an increased column capacity could be advantageous.

For all of the experiments described, we used only CAT12-tag54 unless otherwise stated. At each purification step (load, flow through, wash and elution), samples were analyzed via SDS-PAGE and immunoblotting to determine the presence, concentration, purity and integrity of the CAT12-tag54 (Fig. 3).

On the SDS-PAGE gel and on the immunoblot that used mAb54 as the primary antibody, the elution fraction showed one major band of the expected size of $26 \mathrm{kDa}$ (CAT: $25 \mathrm{kDa}, 12 \operatorname{tag} 54: 1 \mathrm{kDa})$. Apart from the expected band, only one weaker band at $52 \mathrm{kDa}$ was visible. Because this band was detected on the immunoblot, a Coomassie stained gel, a silver stained gel and was furthermore also observed in a commercial CAT protein standard (data not shown), we assumed it to be a CAT dimer. SDS-PAGE and immunoblot analysis also demonstrated high purification efficiency. As no CAT-tag54 was detectable in the flow through or wash fractions, it was evident that all of the available CAT-tag54 in the TSP was adsorbed onto the immunoaffinity matrix and not released until elution.

This a clear advantage of immunoaffinity chromatography systems compared to frequently used purification tags like the His6-tag as the later often leads to a co-purification of host-cell proteins [3]. No contamination with nonspecific 

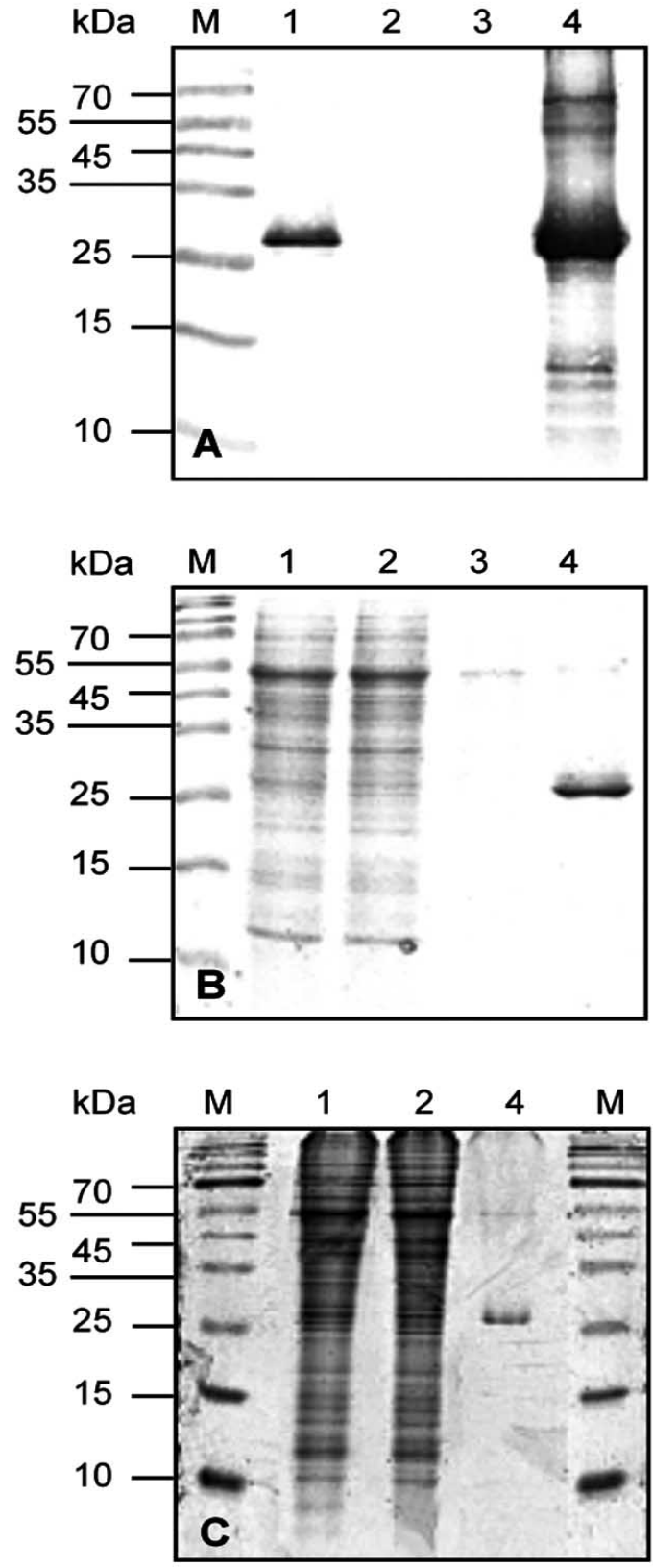

Fig. (3). Immunoaffinity purification of CAT12-tag54.

An extract from transiently transformed tobacco leaves was loaded onto the immunoaffinity matrix. The samples from the purification were gel electrophoresed using a 15\% (w/v) SDS gel. (A) An immunoblot, (B) the gel stained with Coomassie blue, and (C) the silver-stained gel. The plant extract (lane 1), flow through (lane 2), wash (lane 3), and elution (lane 4). Either $6 \mu 1$ (A and B) or $1 \mu 1$ (C) sample were loaded. M: PageRuler protein marker (Fermentas).

or degradation products could be detected in the elution fractions, demonstrating the high purity of the eluted protein. The purified protein was detectable with both the CATspecific antiserum and the mAb54, indicating that integrity of the CAT-tag54 was conserved during the purification procedure. To determine the purification efficiency and yield, the total and native amounts of CAT12-tag54 were quantified in the load, flow through, wash and elution fractions using immunoblotting and ELISA (Fig. 4). The average yield was approximately $100 \%$, but only $11 \%$ of the eluted protein could be detected in its native form. This discrepancy between the native and total protein recovery is caused by the acidic elution conditions. To increase the amount of native eluted protein, we used CAT6-tag54 for purification, assuming that the lower affinity of the 6-tag54 peptide compared to 8- and 12-tag54 peptides as measured by SPR would result in a lower binding strength between the antibody and antigen. In our experiments, however, the lower affinity of the 6-tag54 to the antibody did not lead to elution of CAT6-tag54 under milder conditions (data not shown).
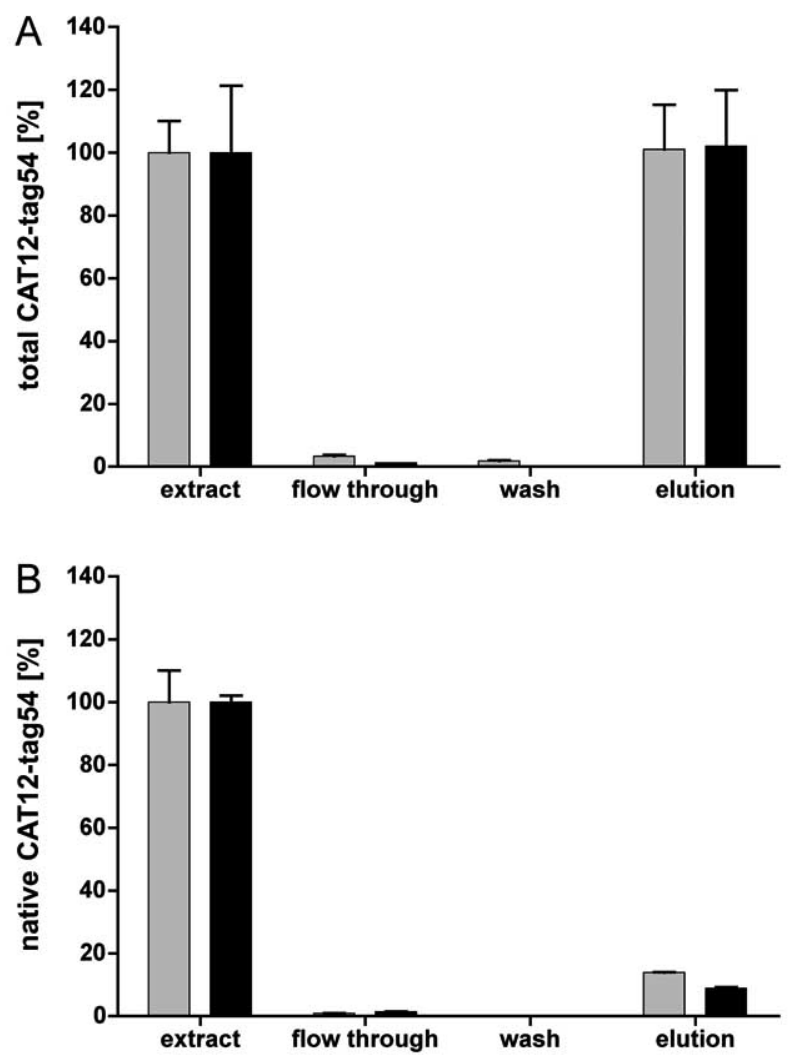

Fig. (4). Purification efficiency of the immunoaffinity chromatography using mAb54/CAT12-tag54.

Two independent purifications, each performed with freshly prepared tobacco leaf extracts, were evaluated and the purification efficiency was measured using immunoblots (A) and ELISA (B). All values are given as percentage of the total amount of CAT12tag54 quantified in the extract (the amount of CAT-12tag54 determined in the extract and elution fractions: experiment I: $153 \pm$ $23 \mu \mathrm{g}$, experiment II: $521 \pm 46 \mu \mathrm{g}$ ). The standard deviation was calculated from six technical replicates for each quantification.

Experiment I: $\square$ Experiment II:

As different proteins have different characteristics, it is likely that other target proteins would maintain their functionality through the immunoaffinity chromatography procedure. Alternatively, instead of an acidic elution, the free tag54 peptide could be the used as competitor as described for the FLAG tag [19]. Also the introduction of a protease cleavage side between the target protein and the tag [20], 
which would allow the release of the target protein under native conditions by on-column cleavage using a specific protease could be an alternative. But this procedure requires an additional purification step to remove the protease - and this will increase the complicity of the purification and it is therefore not the first choice for an alteration.

\section{CONCLUSION}

We have demonstrated the potential of a novel epitope tagging system for the detection and purification of recombinant proteins that are expressed in plants. We were able to detect the tagged reporter protein CAT in the native as well as in the denatured form with the same specificity and sensitivity. We used immunoaffinity chromatography to purify the tagged protein from tobacco leaf extracts in a single step. Using this system, we could recover all of the tagged protein without any detectable contamination. However, purification conditions have to be optimized to improve the purification of sensitive proteins like the CAT enzyme.

\section{ACKNOWLEDGEMENTS}

We would like to thank Dr. Jörg M. Nähring, who prepared the mAb54, and Dr. Stephan Hellwig, for providing the purified antibody. We also thank Holger Spiegel and Markus Sack for support with the surface plasmon resonance analysis.

\section{REFERENCES}

[1] Hochuli E, Dobeli H, Schacher A. New metal chelate adsorbent selective for proteins and peptides containing neighboring histidine-residues. J Chromatogr 1987; 411: 177-84.

[2] Brizzard B. Epitope tagging. Biotechniques 2008; 44(5): 693-5.

[3] Lichty JJ, Malecki JL, Agnew HD, Michelson-Horowitz DJ, Tan S. Comparison of affinity tags for protein purification. Protein Expr Purif 2005; 41(1): 98-105.

[4] Terpe K. Overview of tag protein fusions: from molecular and biochemical fundamentals to commercial systems. Appl Microbiol Biotechnol 2003; 60(5): 523-33.

[5] Boyes DC, Nam J, Dangl JL. The Arabidopsis thaliana RPM1 disease resistance gene product is a peripheral plasma membrane protein that is degraded coincident with the hypersensitive response. Proceedings of the National Academy of Sciences of the United States of America. 1998; 95(26): 15849-54.

[6] Korth KL, Jaggard DAW, Dixon RA. Developmental and lightregulated post-translational control of 3-hydroxy-3-methylglutarylCoA reductase levels in potato. Plant J 2000; 23(4): 507-16.
[7] Kovtun Y, Chiu WL, Zeng WK, Sheen J. Suppression of auxin signal transduction by a MAPK cascade in higher plants. Nature 1998; 395(6703): 716-20.

[8] Naehring J. Herstellung und Expression von"single-chain" Antikörpern gegen das 30K Transport Protein und das 54K Protein von Tobacco Mosaic Virus in Nicotiana tabacum [Dissertation]. Aachen: RWTH Aachen University; 1999.

[9] Felici F, Castagnoli L, Musacchio A, Jappelli R, Cesareni G. Selection of Antibody Ligands from a Large Library of Oligopeptides Expressed on a Multivalent Exposition Vector. J Mol Biol 1991; 222(2): 301-10.

[10] Luzzago A, Felici F, Tramontano A, Pessi A, Cortese R. Mimicking of Discontinuous Epitopes by Phage-Displayed Peptides .1. Epitope Mapping of Human H-Ferritin Using a Phage Library of Constrained Peptides. Gene 1993; 128(1): 51-7.

[11] Van Regenmortel MHV, Altschuh D, Chatellier J, et al. Measurement of antigen-antibody interactions with biosensors. J Mol Recognit 1998; 11(1-6): 163-7.

[12] Kapila J, DeRycke R, VanMontagu M, Angenon G. An Agrobacterium-mediated transient gene expression system for intact leaves. Plant Sci 1997; 122: 101-8.

[13] Geysen HM, Meloen RH, Barteling SJ. Use of Peptide-Synthesis to Probe Viral-Antigens for Epitopes to a Resolution of a Single Amino-Acid. Proceedings of the National Academy of Sciences of the United States of America-Biological Sciences. 1984; 81(13): 3998-4002.

[14] Hujer AM, Bethel CR, Bonomo RA. Antibody mapping of the linear epitopes of CMY-2 and SHV-1 beta-lactamases. Antimicrobial Agents and Chemotherapy 2004; 48(10): 3980-8

[15] Kuttner G, Giessmann E, Wessner H, et al. Linker peptide and affinity tag for detection and purification of single-chain Fv fragments. Biotechniques 2004; 36(5): 864-70.

[16] Zhang L, Hernan RN, Brizzard A. Multiple tandem epitope tagging for enhanced detection of protein expressed in mammalian cells. Mol Biotechnol 2001; 19(3): 313-21.

[17] Einhauer A, Jungbauer A. Affinity of the monoclonal antibody M1 directed against the FLAG peptide. J Chromatogr A 2001; 921(1): 25-30.

[18] Wimalasena RL, Wilson GS. Factors Affecting the Specific Activity of Immobilized Antibodies and Their Biologically-Active Fragments. J Chromatogr-Biomed Applicat 1991; 572(1-2): 85102.

[19] Brizzard BL, Chubet RG, Vizard DL. Immunoaffinity purification of Flag(R) epitope-tagged bacterial alkaline-phosphatase using a novel monoclonal-antibody and peptide elution. Biotechniques 1994; 16(4): 730-5.

[20] Burckstummer T, Bennett KL, Preradovic A, et al. An efficient tandem affinity purification procedure for interaction proteomics in mammalian cells. Nature Methods 2006; 3(12): 1013-9.

(C) Rasche et al.; Licensee Bentham Open.

This is an open access article licensed under the terms of the Creative Commons Attribution Non-Commercial License (http://creativecommons.org/licenses/by-nc/3.0/) which permits unrestricted, non-commercial use, distribution and reproduction in any medium, provided the work is properly cited. 